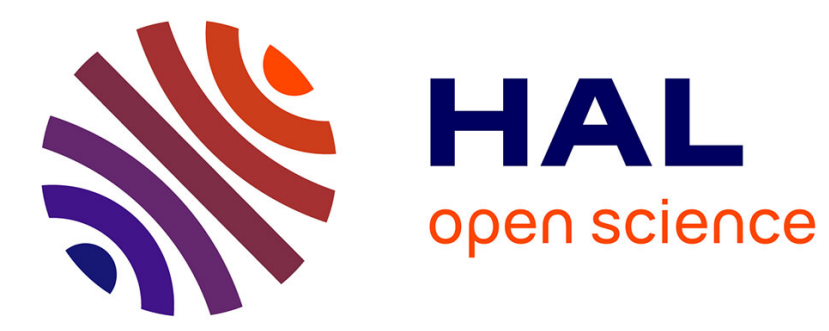

\title{
How to integrate digital post-processing algorithms in professional optical design software for co-designing complex optical systems?
}

\author{
Alice Fontbonne, Hervé Sauer, François Goudail
}

\section{To cite this version:}

Alice Fontbonne, Hervé Sauer, François Goudail. How to integrate digital post-processing algorithms in professional optical design software for co-designing complex optical systems?. Optical Design and Engineering VIII, 118710P, Sep 2021, Madrid, Spain. 10.1117/12.2597059 . hal-03354082

\author{
HAL Id: hal-03354082 \\ https://hal.science/hal-03354082
}

Submitted on 24 Sep 2021

HAL is a multi-disciplinary open access archive for the deposit and dissemination of scientific research documents, whether they are published or not. The documents may come from teaching and research institutions in France or abroad, or from public or private research centers.
L'archive ouverte pluridisciplinaire HAL, est destinée au dépôt et à la diffusion de documents scientifiques de niveau recherche, publiés ou non, émanant des établissements d'enseignement et de recherche français ou étrangers, des laboratoires publics ou privés. 


\title{
How to integrate digital post-processing algorithms in professional optical design software for co-designing complex optical systems?
}

\author{
Alice Fontbonne, Hervé Sauer, and François Goudail \\ Université Paris-Saclay, Institut d'Optique Graduate School, CNRS, Laboratoire Charles \\ Fabry, 91127, Palaiseau, France
}

\begin{abstract}
We investigate hybrid imaging systems consisting of a lens and a fast post-processing algorithm, where the lens is designed or co-designed with professional systems optical design software. Two approaches have been investigated: adapt the optimization criterion to the use of a unique deconvolution filter by requiring uniformity of the modulation transfer functions (MTF) over the field of view, or use piecewise-constant deconvolution to adapt the processing algorithm to spatial variations of the MTF in conventionally designed optical systems. By studying the evolution of the deconvolution performance across the field of view in systems optimized with these two approaches, we highlight that the optimal use of post-processing algorithm leads to lenses whose intrinsic performance varies little in the field of view, with however a significant amount of appropriate aperture aberrations but well suited to be post-processed.
\end{abstract}

Keywords: Image processing, piecewise-constant deconvolution, optical co-design, hybrid imaging system

This pre-print has been published as: Alice Fontbonne, Hervé Sauer, François Goudail, "How to integrate digital postprocessing algorithms in professional optical design software for co-designing complex optical systems?" Proc. SPIE 11871, Optical Design and Engineering VIII, 118710P (12 September 2021)

DOI: https://doi.org/10.1117/12.2597059.

\section{INTRODUCTION}

Today, almost all imaging systems include both an optical part (the lens) and an image processing part in order to improve the final image quality. It therefore seems natural to optimize them simultaneously to obtain the best possible result. However, even if this "co-design" approach is more and more recognized at a conceptual level, it is still rarely used in practice for designing complex lenses with many adjustable parameters and constraints. This is due to the fact that the contribution of image processing is currently difficult to take into account in optical systems design software.

Until now, the field of co-design has thus mainly focused on simplest imaging systems, consisting for example of single co-optimized optical elements such as phase masks. " One of the first attempts to co-design optical systems involving complex lenses has been done by Stork \& Robinson, ${ }^{?}$ who integrated the image processingbased criterion known as mean square error (MSE) in the optical software Zemax OpticStudio. It is also possible to consider other optimization criteria than the MSE, such as a surrogate criterion built from more classical optical parameters (modulation transfer function, point spread function, etc.)?

In the present paper, we investigate and compare two optimization criteria for designing hybrid systems with professional optical systems design software: a conventional criterion, based on minimizing the spread of spot diagrams, and a "codesign-based" criterion, that enforces small changes of the MTF over the field of view. The images obtained from the systems optimized with these two criteria can be post-processed with a 
single deconvolution filter, or with a piecewise-constant deconvolution algorithm. We study the impact of the optimization criterion and of the post-processing algorithm on the global performance of these different systems. This article is organized as follow: firstly, we define in detail the two optimization criteria for hybrid systems (Section 2) and then we evaluate the performance of the designed systems when the image is post-processed with a piecewise-constant deconvolution algorithm of adjustable number of zones, including one, i.e. the single filter case (Section 3). Finally, we conclude in Section 4 about these different approaches to design a hybrid system with a professional optical systems design software and to use it in practice.

\section{DESIGN HYBRID IMAGING SYSTEMS IN PROFESSIONAL OPTICAL SYSTEMS DESIGN SOFTWARE}

\subsection{Specifications and goal}

We consider hybrid optical-digital systems composed of two main parts: an imaging system (i.e. a lens and a sensor) and an image processing algorithm consisting of a deconvolution filter. The goal of the design (or codesign) in this paper is to obtain a hybrid system with the best mean performance possible for all the positions over the field of view. Both hybrid systems (the conventional and the codesigned one) are optimized with the same specifications. First, we consider a F/4 lens of $50 \mathrm{~mm}$ focal length made of two cemented doublets separated by a diaphragm. Limitations on the size of the lens are the same for the two systems. Secondly, the processing algorithm is the same of both systems. We use a fast algorithm that take approximately the same computation time as a single convolution.

By considering a set of $K$ differents positions in the field $\Psi=\left\{\psi_{1}, \psi_{2}, \ldots \psi_{K}\right\}$ (in ascending order), it as been established $^{\text {? }}$ that the best linear filter to deconvolve an image is the averaged Wiener filter

$$
\tilde{w}(\nu)=\frac{\frac{1}{K} \sum_{k} \tilde{h}_{\psi_{k}}(\nu)^{\star}}{\frac{1}{K} \sum_{k}\left|\tilde{h}_{\psi_{k}}(\nu)\right|^{2}+\frac{S_{n n}(\nu)}{S_{o o}(\nu)}},
$$

where $\tilde{h}_{\psi_{k}}(\nu)$ is the local optical transfer function (OTF) at the position $\psi_{k}$ in the field of view $\left(\tilde{h}_{\psi_{k}}\right.$ being the Fourier Transform of the PSF $h_{\psi_{k}}$ ), * stands for the complex conjugate, $S_{n n}(\nu)$ is the power spectral density (PSD) of the noise and $S_{o o}(\nu)$ is the PSD of the scene. Note that for the sake of simplicity in the formulae, we use the symbol $\nu$ for the $2 \mathrm{D}$ spatial frequency vector $\left(\nu_{x}, \nu_{y}\right)$. We consider a power-law object PSD $S_{o o}(\nu) \propto\|\nu\| \|^{-2.5}$ and a white noise PSD such that the signal-to-noise ratio (SNR) is equal to $34 \mathrm{~dB}$. It corresponds to a standard digital camera under good illumination conditions.

This averaged Wiener filter is applied to the image provided by the imaging system. We consider two systems: a conventional one (Section 2.2) and a system co-optimized in a professional optical systems design software by taking into account the impact of the deconvolution on the final performance (Section 2.3).

\subsection{Conventionally optimized hybrid imaging system}

There are several ways to optimize a lens with professional optical systems design software. The most usual way is to minimize the quadratic sum of the root mean square (RMS) diameters of the spot diagrams for all the considered field positions. This method, which does not take into account any post-processing algorithm, allows to limit the aberrations of an optical system so that the spot diagrams are, on quadratic average, as small as possible. Even if the modulation transfer functions (MTF) are often used as a tool by the optical designer to check the performance of the system, we do not directly consider them in the optimization criterion.

Let us consider for example an optimization, with this criterion, of a lens made of two cemented doublets separated by a diaphragm. Figure 1(a) shows the spot diagrams (extracted from Synopsys CodeV) of the optimized system at four positions in the field, from the on axis position (bottom of the figure) to the maximum object field position, of $20^{\circ}$. We can notice that the conventional optimization led to spot diagrams whose sizes vary considerably over the field of view. Small sizes occur at positions in the field which are close to the axis. However, on the axis, the width of the spot is more important, due to curvature of field and best composite focusing. The spot diagram for the maximum position in the field is the largest: its RMS width is almost twice 
(a)

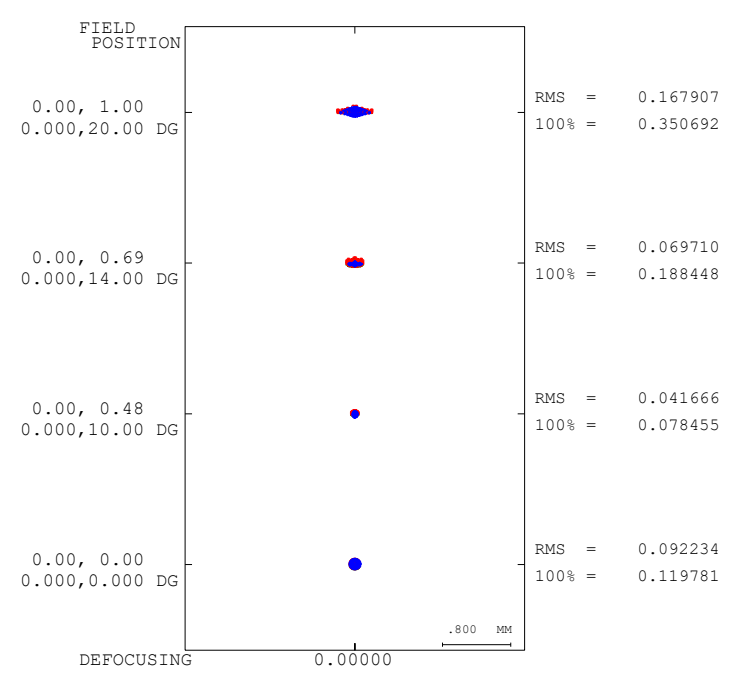

(b)

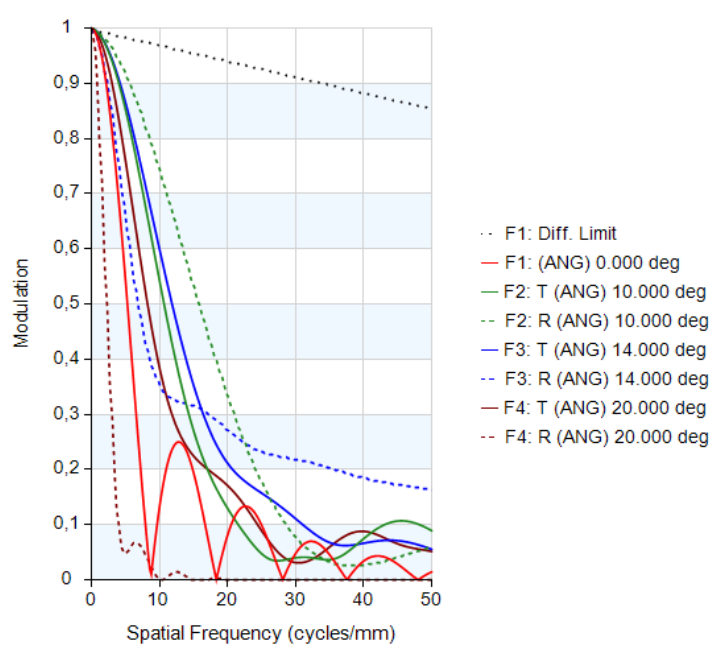

Figure 1. (a) Spot diagrams of the conventional optical system (b) MTFs of the conventional optical system.

the one of the on axis spot diagram, and almost four times that of the smallest spot diagram among the four plotted on Figure 1(a).

This variation in the sizes of the spot diagrams of course induces a variation of the MTFs, Figure 1(b). The smallest spot diagrams, obtained for the $10^{\circ}$ and $14^{\circ}$ fields, give the highest MTF for both sagittal and tangential orientations. The larger spot diagram on the axis causes the corresponding MTF to show successive nullings, starting from $9 \mathrm{lp} . \mathrm{mm}^{-1}$. Finally, the sagittal MTF of the maximum position in the field $\left(20^{\circ}\right)$ is nearly equal to zero starting from $10 \mathrm{lp} \cdot \mathrm{mm}^{-1}$, which is linked to the fact that the corresponding spot diagram is very large along the horizontal direction.

The evolution of the shape of the spot diagrams of this system (Figure 1(a)) and its MTF (Figure 1(b)) is usual in conventional optical design. This optical system is not co-designed, but it is still possible to add digital post-processing such as the averaged Wiener filter (Eq. 1), so that its performance can be compared with that of a co-designed hybrid system.

\subsection{Co-optimized hybrid system}

The idea of co-design is to design, in the same time, the optical system and the associated digital processing (for this article, the averaged Wiener filter calculated from the OTFs of the optical system). As for any optimization, a criterion to be minimized must be defined. The averaged Wiener filter (Eq. 1), built with a set of OTFs calculated for a representative set of positions in the field $\Psi$ (the set $\Psi$ comprising $\mathrm{K}$ positions in the field) is the linear filter allowing to minimize the MSE criterion, defined in the Fourier space by:?

$$
M S E(\Psi)=\frac{1}{K} \sum_{k} \int_{\nu}\left[S_{o o}(\nu)\left|\tilde{h}_{\psi_{k}}(\nu) \tilde{w}(\nu)-1\right|^{2}+|\tilde{w}(\nu)|^{2} S_{n n}(\nu)\right] d \nu
$$

This optimization criterion is not natively implemented in professional optical systems design software such as Zemax or CodeV. A way around this problem has already been proposed in Zemax, ${ }^{?}$ but until now, no publication, to the best of our knowledge, claims to have implemented it in CodeV, the software we use here. Instead, a surrogate criterion to MSE has been proposed.? It is a compound criterion taking into account both the size of the spot diagrams (like the conventional criterion) and the disparity between the MTFs over the field of view. Indeed, as a single deconvolution filter is used, the optimization on the MSE criterion naturally leads 
to the approximation that the MTFs have to be close to each other for all the positions in the field. ${ }^{?}$ It is this behavior that is reproduced with the implementation of such a surrogate criterion.

In this article, we use a similar algorithm which consists in equalizing the MTFs by constraining their value differences to zero at two or three spatial frequency values. It is possible to change the values of the frequencies and constraints during the design path, to facilitate equalization. Note that, with this method, only the MTFs are optimized and not the spot diagrams. Therefore, we had to constrain the level of the MTFs in addition to equalizing them so that they are not too low (because the size of the spot diagrams is not minimized).

The MTFs of the system optimized with this criterion are given in Figure 2(b). As expected, they are quite

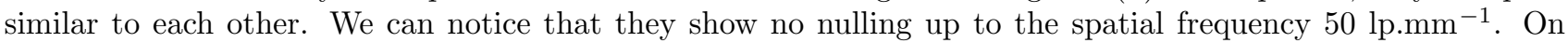
the other hand, they are all rather weak $(<0.05)$ at this frequency, which was not the case for the conventional system. They drop very sharply up to $5 \mathrm{lp} . \mathrm{mm}^{-1}$ but, starting from this frequency (where all MTFs are about 0.3 ), the decay slope becomes relatively small. Optimizing on MTFs equalization is very interesting when using a single deconvolution filter as the hybrid system behavior is independent of the position in the field of view. It is also important that there is no nulling, because a nulling of the MTFs causes a loss of information that cannot be recovered by deconvolution. However, as the co-optimized MTFs are quite low $(<0.25$ starting from $10 \mathrm{lp} \cdot \mathrm{mm}^{-1}$ ), the reconstruction can be difficult if there is too much noise. We have considered from the beginning the case of a good SNR, which is favorable to this method of co-design by MTFs equalization.

(a)

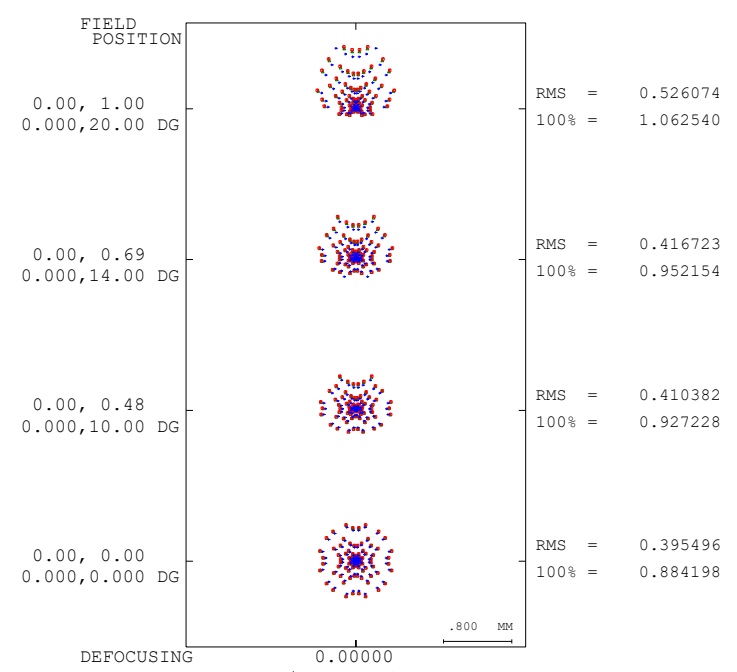

(b)

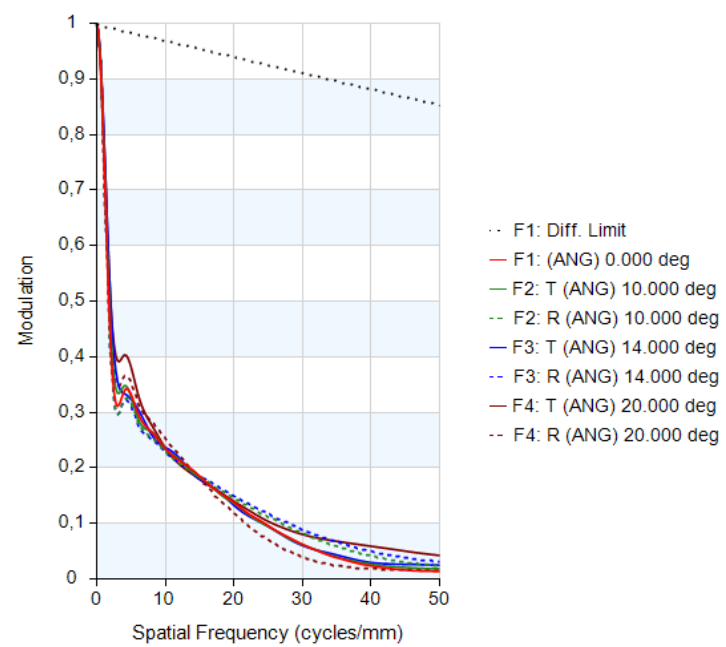

Figure 2. (a) Spot diagrams of the co-designed optical system (b) MTFs of the co-designed optical system.

Let us now examine, Figure 2(a), the spot diagrams of this system co-designed by MTFs equalization. They are similar to each other, and are very large compared to those of the conventional system (the scale is the same for Figure 1(a) and Figure 2(a)). Globally, the RMS size of spot diagrams in the co-designed case is five to ten times larger than in the conventional case. This is an important difference between the conventional optimization method and the presented co-optimization method. Such large spot diagrams are typical of an imaging system corrupted by strong aperture aberrations, which is bad when used without digital post-processing, but its images can be efficiently restored with a unique deconvolution filter.

To conclude, we have obtained two imaging systems that can be used with digital post-processing. The first one, optimized in a conventional way, gives priority to the small size of the spot diagrams. However, as all its MTFs are different from each other, it is not very well suited for use with a single deconvolution filter. The second one is co-optimized by equalizing the MTFs in view of its use with an averaged Wiener filter. In the following section 3, we will evaluate the performance after post-processing of the two hybrid systems. 


\section{PIECEWISE-CONSTANT DECONVOLUTION FOR HYBRID SYSTEMS}

In the previous section, the co-designed system has been optimized by enforcing similarity of the MTFs over the field of view. Deconvolution with a unique averaged Wiener filter is thus an appropriate post-processing algorithm for this system. In contrast, it is not appropriate for the optical system optimized in a conventional way since its MTFs vary significantly in the field of view. Hence, we will also consider in this section another post-processing algorithm that takes into account MTF variation over the field. Reconstruction algorithms for continuously varying blur exist, ${ }^{\text {? }}$ but in this paper, we will only consider non-recursive piecewise-constant deconvolution algorithms whose advantage is to keep the computational time equivalent to that of a single convolution.

\subsection{Evolution of the MSE with piecewise-constant deconvolution}

We propose here to use a method of piecewise-constant deconvolution. It means the entire field of view is divided into square areas and, for each area, a deconvolution by an averaged Wiener filter is performed. The used filter depends on the area since each filter is built from a set of $\Psi_{A}$ positions in this area $A$. Therefore, for each area $A$, it is possible to calculate the local value of the MSE, $M S E\left(\Psi_{A}\right)$, by using the Eq. 2. The total MSE for the entire field of view of the lens is the mean of the MSE of the different areas (of equal size):

$$
\operatorname{MSE}\left(\Psi_{t o t}, N_{A}\right)=\frac{1}{N_{A}} \sum_{A} M S E\left(\Psi_{A}\right)
$$

where $\Psi_{t o t}$ denotes the set of positions in the field for the entire field of view and $N_{A}$ is the number of areas considered, such as $\cup_{A} \Psi_{A}=\Psi_{\text {tot }}$.

This section aims to evaluate how the quality of the deconvolution of hybrid systems evolves with the number of areas considered for the piecewise-constant deconvolution $\left(N_{A}=1\right.$ gives the unique averaged Wiener filter). All simulations will be done considering a sensor of Nyquist frequency $100 \mathrm{lp} . \mathrm{mm}^{-1}$. Note that, instead of the $\operatorname{MSE}\left(\Psi_{t o t}\right)$, we will prefer to use the mean image quality as a performance metric for hybrid systems (expressed in $\mathrm{dB})$ :

$$
I Q_{\text {mean }}\left(N_{A}\right)=10 \log _{10} \frac{\sigma_{O}^{2}}{M S E\left(\Psi_{t o t}, N_{A}\right)} .
$$

where $\sigma_{O}^{2}$ denotes the variance of the sharp image.

First, it is possible to see on Figure 3 that the mean performance before deconvolution is far better $(+2.8 \mathrm{~dB})$ for the conventional system than for the co-designed one. This is due to the fact that, on average and except for special cases (on the axis or extreme field), the MTFs of the conventional system (Fig. 1) are higher than those of the co-designed system (Fig. 2). After deconvolution with a unique averaged Wiener filter $\left(N_{A}=1\right)$, the two systems have very equivalent performance, close to $10 \mathrm{~dB}$. This represents a significant performance gain for the co-designed system $(+3.7 \mathrm{~dB})$, showing that the MTFs equalization criterion is very well suited for use with a single deconvolution filter. On the other hand, the gain is less important for the conventional system, only $0.9 \mathrm{~dB}$ : the strong variations of the MTFs across the field prevent the single deconvolution from working well.

For the conventional hybrid system, when the number of areas $N_{A}$ increases, $I Q_{\text {mean }}$ is slowly increases. It reaches $11.2 \mathrm{~dB}$ for $N_{A}=25$ areas, i.e. when the field is divided into $5 \times 5$ equal areas. On the contrary, for the co-designed system, $I Q_{\text {mean }}$ sharply increases (of $2 \mathrm{~dB}$ when $N_{A}=4$ ), and then tends towards an asymptote. It reaches $13.3 \mathrm{~dB}$ for $N_{A}=25$ areas. Thus, the conventional system and the hybrid system have a quite different behavior regarding the number of areas. This discrepancy can be explained by the differences of MTF variations with the position in the field, as will be shown in the following.

\subsection{Disparity between MTFs and intrinsic difficulty of deconvolution}

The MSE of an area $A$ depends on the values of the OTFs $\tilde{h}_{\psi}(\nu)$ over this area. To facilitate the interpretation of the evolution of the MSE with the field of view, it is possible to split the expression of the MSE (Eq. 1), 


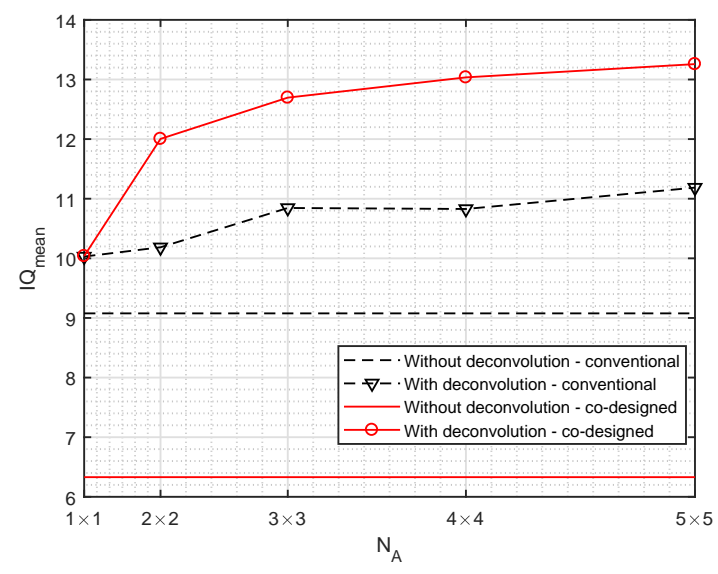

Figure 3. $I Q_{\text {mean }}$ as a function of the number of areas $N_{A}$ considered for the piecewise-constant deconvolution, for the conventional system and the co-designed system. Note that $N_{A}=1$ gives the post-processing with the unique averaged Wiener filter.

corresponding to a set of positions $\Psi$ in the field of view, into two parts? $M S E_{D}$ and $M S E_{I}$, so that $M S E(\Psi)=$ $M S E_{D}(\Psi)+M S E_{I}(\Psi)$.

The first part,

$$
M S E_{D}(\Psi)=\int_{\nu} \frac{S_{o o}(\nu)^{2}\left[\frac{1}{K} \sum_{k}\left|\tilde{h}_{\psi_{k}}(\nu)\right|^{2}-\left|\frac{1}{K} \sum_{k} \tilde{h}_{\psi_{k}}(\nu)\right|^{2}\right]}{S_{o o}(\nu) \frac{1}{K} \sum_{k}\left|\tilde{h}_{\psi_{k}}(\nu)\right|^{2}+S_{n n}(\nu)} d \nu
$$

is characteristic of the disparity between the MTFs, and would be null if all the OTFs were equal across the field of view. Considering an area $A$, the more the local OTF varies with the position in the field (in $\Psi_{A}$ ), the more $M S E_{D}\left(\Psi_{A}\right)$ will be high.

The other term is

$$
M S E_{I}(\Psi)=\int_{\nu} \frac{S_{o o}(\nu) S_{n n}(\nu)}{S_{o o}(\nu) \frac{1}{K} \sum_{k}\left|\tilde{h}_{\psi_{k}}(\nu)\right|^{2}+S_{n n}(\nu)} d \nu
$$

which is equal to $M S E(\Psi)$ if all the OTFs at all the positions in the field of view are equal. Therefore, this term is characteristic of the intrinsic difficulty of deconvolution, i.e. of the compromise made by the averaged Wiener filter between noise amplification and signal reconstruction with the average MTF $\sqrt{\frac{1}{K} \sum_{k}\left|\tilde{h}_{\psi_{k}}(\nu)\right|^{2}}$.

\subsubsection{Evolution of area-specific MSE with the number of areas for the conventionally optimized system}

Figure 4 represents the piecewise-constant square root of $M S E_{D}\left(\Psi_{A}\right), M S E_{I}\left(\Psi_{A}\right)$ and $M S E\left(\Psi_{A}\right)$ for the conventional system and different divisions in the field of view. These divisions range from 1 (using a single deconvolution filter for the entire field) in the first row to 25 (using then 25 different averaged Wiener filters for $5 \times 5$ areas of equal size) in the last row.

We can first notice on Fig. 4 that, as the number of areas $N_{A}$ increases, the intrinsic difficulty of deconvolution (column a) increases for some areas. It remains constant with the increase in $N_{A}$ only for the areas located in the region corresponding to spot diagrams of small diameters, that is, around $10^{\circ}$ (see Fig. 1(a)). Secondly, the contribution of the disparity between the MTFs (Fig. 4(b)) decreases with the number of areas but in a way depending on the location of the area in the field. $M S E_{D}$ remains approximately constant with the number of areas $N_{A}$ in the areas of strong radial or azimuthal variations of MTFs and decreases for the other areas. It follows from the sum of these two contributions that the square root of the MSE (RMSE, Fig. 4(c)) globally decreases (with the number of areas) for the areas where the disparity between the MTFs decreases. However, it increases for the other areas, because the disparity between the MTFs has remained stable while the intrinsic difficulty of deconvolution increases since the spot diagrams in the field are wider. 


\subsubsection{Evolution of area-specific MSE with the number of areas for the co-optimized system}

Figure 5 shows that the behavior of the co-optimized hybrid system is very different from the previous one. The first thing to note is that the intrinsic difficulty of deconvolution remains almost constant, regardless of the number of areas. This is due to the fact that the co-designed system has a very homogeneous performance in the field of view (see Fig. 2). However, the value of $M S E_{I}\left(\Psi_{A}\right)$ is larger on average than for the conventional system, since the average MTF of the co-designed system is quite low.

We can also observe that, as the number of areas increases, the RMSE related to the disparity between the MTFs (Figure 5(b)) approaches zero, as the closer the positions considered in the field are, the closer the MTFs are to each other. The extreme field area has the highest RMSE because the extreme field spot diagrams does no more exhibit a circular symmetry (Fig. 2(a)), which leads to a larger disparity between the different local MTFs in the area.

Therefore, the total RMSE (Fig. 5(c)) essentially follows the improvement due to the increasingly strong match between MTFs with the increase in the number of areas. This explains the rapid increase of $I Q_{\text {mean }}\left(N_{A}\right)$ (Fig. 3) for the co-designed system by MTFs equalization, and also the fact that there is an asymptote: $M S E_{I}\left(\Psi_{A}\right)$ is stable and $M S E_{D}\left(\Psi_{A}\right)$ cannot be smaller than 0 .

As a general conclusion on the studied systems, the error due to the intrinsic difficulty of deconvolution tends to increase with the number of areas used to divide the field of view. This observation is particularly relevant for the conventional system, for which the spot diagram varies (and often grows) in the field of view, causing an increase in the associated intrinsic difficulty of deconvolution. In contrast, by construction, codesigned systems are little affected by this increase. On the other hand, the disparity between MTFs tends to decrease with the number of areas (i.e., as the areas become smaller), except in areas of rapid azimuthal or radial variation of the spot diagram. Since areas of strong radial or azimuthal variation are often correlated with the presence of a larger spot diagram (due to strong field aberrations), these areas see their intrinsic difficulty of deconvolution increase with the increase of the number of areas $N_{A}$, whereas the disparity between the MTFs is not decreasing. Therefore, for these areas, the total RMSE increases. If we consider the whole image, this increase is counterbalanced by the decrease of the RMSE in the simpler areas so that the total RMSE continues to decrease with the number of areas, as seen on Fig.3.

\section{CONCLUSION}

Today, it would be possible to implement the standard co-design criterion of MSE in professional optical systems design software such as Zemax and CodeV. However, this approach, difficult to implement, is still rarely used. To optimize a hybrid system, two simple approaches to implement are the conventional approach (optimize on spot-diagram size) and the co-design approach by MTFs equalization.

Fast processing algorithms, such as piecewise-constant deconvolution, can be added to optical systems. We studied the potential gain of this post-processing algorithm on conventionally and co-optimized systems. This study has shown that piecewise-constant deconvolution systematically allows to refine and improve the reconstruction with the increase of the number of considered areas (as they get smaller). This improvement levels off for a large number of areas, in particular for co-designed systems, which shows that in this case, a limited number of areas is sufficient to reach optimal performance. For a fixed number of areas, piecewise-constant deconvolution works better for a slow variation of the MTF in these areas. Therefore, it works well on a co-designed system because the disparity between MTFs is small by construction, but may not be able to compensate for the fast variation of the MTF observed in a conventional system.

To conclude, it is worth taking into account, from the optimization phase of the lens, that a deconvolution will be used. In fact, even if the processing algorithm is adapted as much as possible to shift variant blur, slow variations of the MTF in the field is more appropriate for use with a deconvolution filter. This study remains to be generalized on more complex optical systems or systems co-optimized with the MSE criterion. 


\section{ACKNOWLEDGMENTS}

The work reported in this study is supported in part by the Agence de l'Innovation de Défense (AID) that provides half of a $\mathrm{PhD}$ fellowship to Alice Fontbonne. Authors have no conflicts of interest related to the work leading to the results presented in this paper.

Alice Fontbonne received her MS degree in optics from Institut dOptique Graduate School. She is now a PhD fellow at Institut dOptique Graduate School (Palaiseau, France) and works on joint design of optics and image processing as a new approach to high level optical design.

Hervé Sauer is a former student of the École Normale Supérieure de Fontenay/St-Cloud. He got the Agregation degree in physics in 1985, and a MSc and PhD in physics from Université Paris Sud-XI (Orsay) in 1985 and 1990 respectively. He is an associate professor at Institut d'Optique Graduate School (Palaiseau) since 1990. His main research topics deal with simulations of optical systems and optical system design.

François Goudail graduated from the École Supérieure d'Optique, (Orsay), in 1992 and obtained his PhD in 1997 from the University of Aix-Marseille III. He has been associate professor at Fresnel Institute, (Marseille), until 2005. He is now professor at the Institut dOptique Graduate School (Palaiseau). His research topics include information extraction in images from different types of passive and active sensors (hyperspectral, SAR, polarimetric), wavefront engineering and joint design of optical systems and image processing algorithms. 

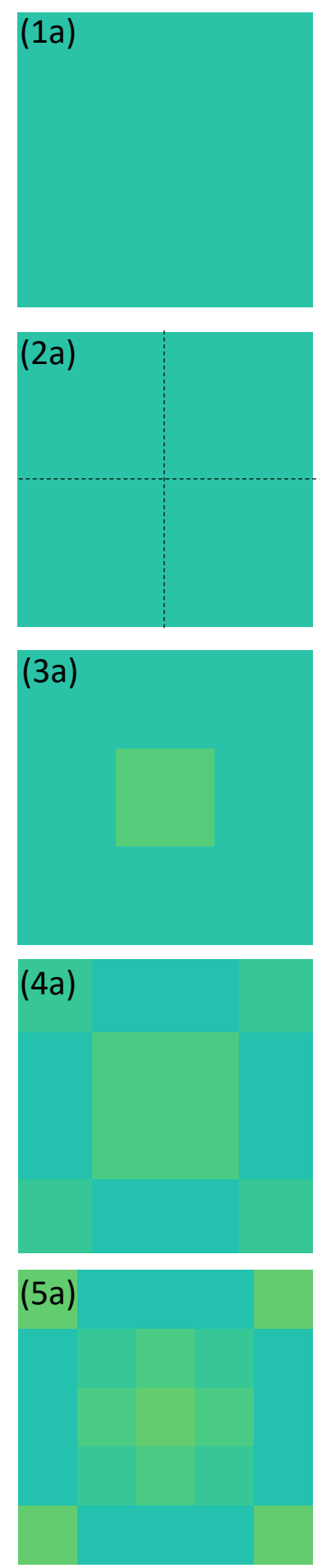
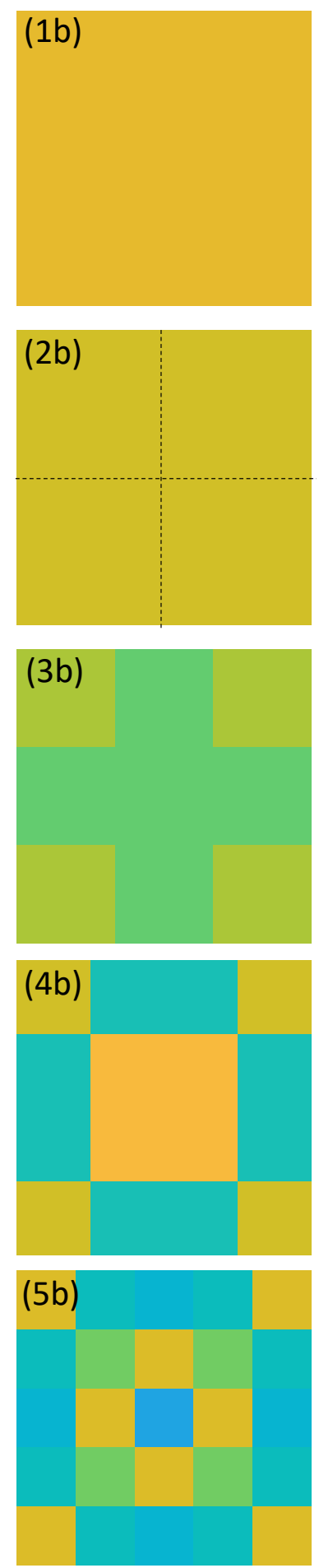
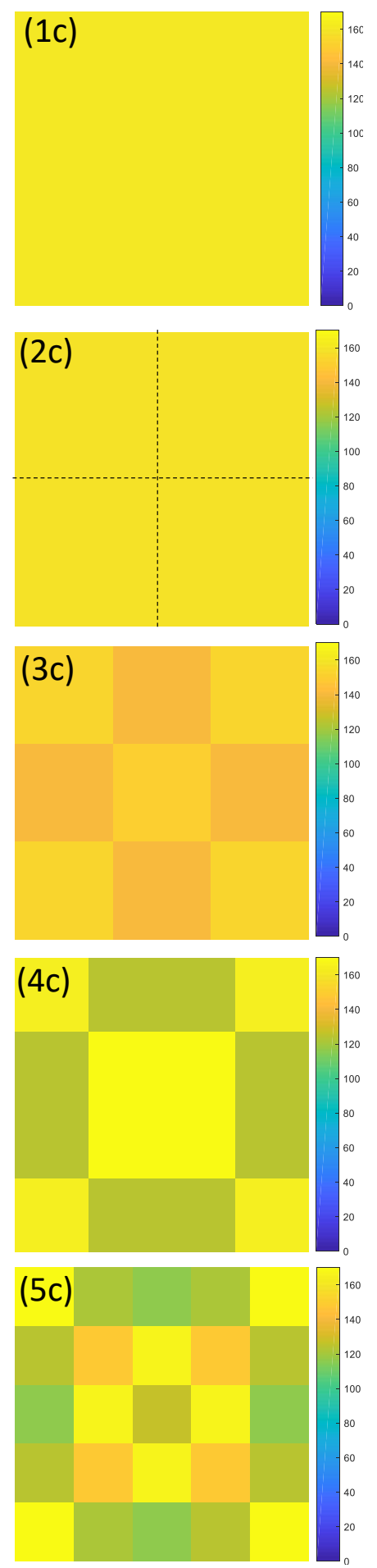

Figure 4. Piecewise-constant RMSE after deconvolution for the conventional hybrid system. (First column) Intrinsic difficulty of deconvolution $\sqrt{M S E_{I}}$; (Second column) Disparity between MTFs $\sqrt{M S E_{D}}$; (Third column) Total $\sqrt{M S E}$. The number of areas used for the piecewise-constant deconvolution depends on the row: (1) one area; (2) four areas; (3) nine areas; (4) sixteen areas; (5) twenty-five areas. The colorbar is the same for all the graphs. 

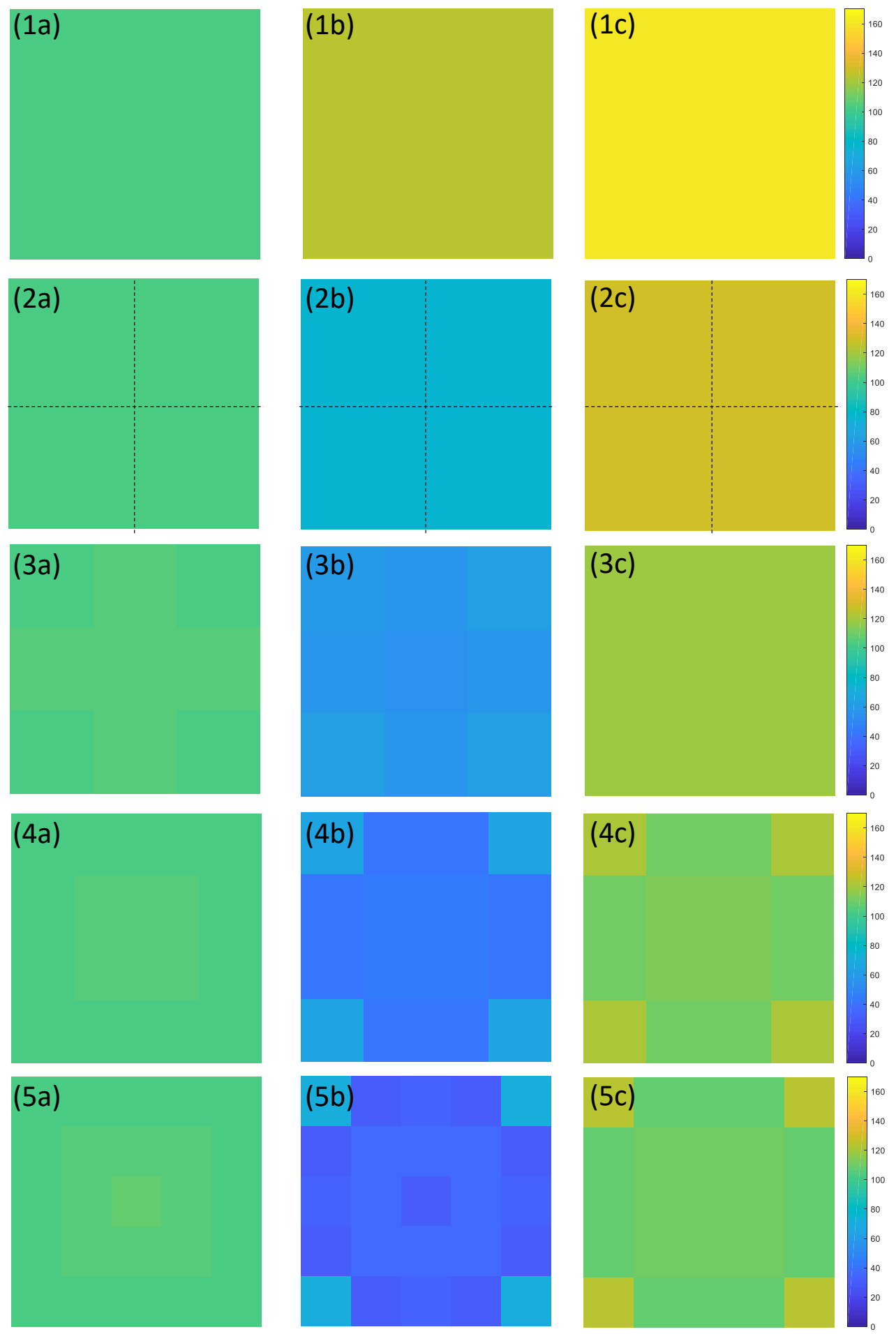

Figure 5. Piecewise-constant RMSE after deconvolution for the co-designed hybrid system. (First column) Intrinsic difficulty of deconvolution $\sqrt{M S E_{I}}$; (Second column) Disparity between MTFs $\sqrt{M S E_{D}}$; (Third column) Total $\sqrt{M S E}$. The number of areas used for the piecewise-constant deconvolution depends on the row: (1) one area; (2) four areas; (3) nine areas; (4) sixteen areas; (5) twenty-five areas. The colorbar is the same for all the graphs. 